\title{
Errata: Properties of Step-Edge Pb-Si-Pb Josephson Junctions*
}

\author{
A. Serfaty, J. Aponte, and M. Octavio \\ Instituto Venezolano de Investigaciones Cientificas, Caracas, Venezuela
}

(Received November 5, 1986)

Equation (1) should read

$$
R_{n}=(\pi / \sigma w)[\ln (8 k / L)]^{-1}
$$

In Eq. (3) the device length $L$ was called $a$ to correspond to the notation of Ref. 15.

In Fig. 4 the critical current is scaled using $J_{c} / B\left(6 k_{\mathrm{B}} / \pi \hbar \tau\right)^{1 / 2}$ and not $J_{c} / B\left(6 k_{\mathrm{B}} / \pi \hbar \tau\right)$ as mentioned in the text.

*This paper appeared in J. Low Temp. Phys. 63, 23 (1986). 